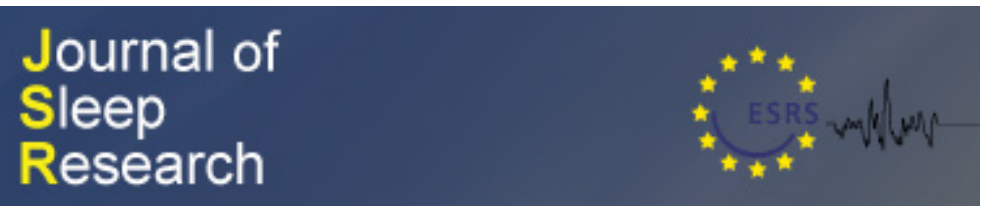

\title{
Adolescent Sleep Patterns are Associated with the Selection of Positive and Negative Emotional Situations
}

\begin{tabular}{|r|l|}
\hline Journal: & Journal of Sleep Research \\
\hline Manuscript ID & JOSR-19-019.R2 \\
\hline Manuscript Type: & Regular Research Paper \\
\hline Date Submitted by the \\
Author: & n/a \\
\hline Complete List of Authors: & $\begin{array}{l}\text { Palmer, Cara; Montana State University, Psychology } \\
\text { Alfano, Candice A.; University of Houston, Department of Psychology } \\
\text { Bower, Joanne; De Montfort University, School of Applied Social Sciences }\end{array}$ \\
\hline Keywords: & emotion regulation, sleep variability, youth, adolescence, reward, mood \\
\hline &
\end{tabular}

\section{SCHOLARONE Manuscripts}


Adolescent sleep patterns are associated with the selection of positive and negative emotional situations

Cara A. Palmer

Assistant Professor, Department of Psychology, Montana State University, Bozeman, Montana, USA

Candice A. Alfano

Professor, Department of Psychology, University of Houston, Houston, Texas, USA

Joanne L. Bower

Lecturer, School of Applied Social Sciences, De Montfort University, Leicester, England

Total Words: 5285

Number of References: 47

Acknowledgements: This research was supported by a grant from the University of Houston.

Conflicts of interest: The authors do not have any conflicts of interest to report.

Author contributions: $\mathrm{CP}, \mathrm{JB}$, and $\mathrm{CA}$ were involved in study design/development and data collection. CP conducted the statistical analyses and prepared the manuscript. CA and JB provided feedback and edits to the manuscript.

\section{Corresponding author:}

Cara A. Palmer, Ph.D.

319 Traphagen Hall

Montana State University

Bozeman, MT 59717

cara.palmer@montana.edu

Phone: 406-994-6052

Fax: 406-994-3804 


\begin{abstract}
Summary
Poor sleep in youth is a risk factor for experiencing increased negative emotions and decreased positive emotions, which can contribute to the development of later emotional disorders.

Understanding of specific processes that produce sleep-related alterations in emotion is limited, although preliminary studies suggest changes in the ability to appropriately regulate or control emotions as one mechanism. The current study builds on this research by examining the relationship between adolescent sleep patterns and a previously unexplored emotion regulation strategy: situation selection. Situation selection strategies are implemented prior to the onset of an emotional experience via decisions to approach rewarding/positive situations or avoid unwanted/negative situations. Fifty-four healthy adolescents (ages 13-17) completed one week of actigraphy and assessments of situation selection using: 1) trait-based questionnaires, 2) daily reports, and 3) an experimental lab task where participants were given the option to watch various emotional video clips of their choice. Greater variability in sleep timing was associated with less avoidance of negative emotional situations, and a longer sleep onset latency was associated with more avoidance of negative emotional situations. Greater variability in nightly sleep patterns was also associated with decreased tendencies to select positive emotional situations as assessed by trait questionnaires, daily reports, and the lab-based task, but only for boys. These findings add to a growing body of research on sleep and emotional experience and provide further support for the importance of intraindividual variability of sleep patterns in youth.
\end{abstract}

Keywords: emotion regulation, sleep variability, youth, adolescence, reward, mood 
Unhealthy sleep patterns are common among adolescents and are increasing in prevalence, with up to $75 \%$ of teens obtaining insufficient sleep (Keyes et al., 2015; Owens et al., 2014). While acute poor sleep results in degraded functioning across a broad range of domains, persistent sleep problems heighten future risk for the development of psychiatric disorders such as depression (e.g., Greene et al., 2015). Available research suggests that emotional processes may be one mechanism by which poor sleep increases psychiatric risk (Harvey et al., 2011; Palmer et al., 2018; Reddy et al., 2017). This is supported by experimental studies showing loss of sleep to produce maladaptive emotional experiences, such as increased negative affect and decreased positive affect (Palmer and Alfano, 2017). This may be particularly pertinent for youth, who show greater sleep-based emotional alteration compared to adults (e.g., McGlinchey et al., 2011; Talbot et al., 2010), presumably due to the developing maturity of the pre-frontal cortex (PFC), which is critical for regulating affective responses (Casey et al., 2008). Despite this, discrete processes underlying emotional alterations are still poorly understood, particularly for youth samples. The current study builds on existing research by examining the relationship between adolescent sleep and a previously unexplored emotion regulation strategy: situation selection.

In order to experience an emotion, an individual must first encounter an emotional situation, attend to and appraise that situation as negative or positive, which ultimately generates an emotional response. The process model of emotion regulation (Gross, 1998) suggests that the experience of an emotion can be altered or regulated at any of these stages of the emotion generation process (e.g., changing the focus of one's attention or reappraising the situation). Research suggests that adolescent sleep disruption is associated with more overall emotion dysregulation (Baum et al., 2014) and more suppression of emotional expressions, rumination, avoidance, and less problem solving when faced with a social stressor (e.g., Palmer et al., 2018). 
Emotion regulation strategies can also be implemented prior to the onset of an emotional experience, before an emotion occurs, by making decisions to approach rewarding situations or avoid unwanted emotional situations. These antecedent-focused emotion regulation strategies have been referred to as situation selection (Gross, 1998). Situation selection may be a particularly effective means to control emotional experiences because it minimizes undesirable emotional reactions requiring regulation (Duckworth et al., 2016), and thus is generally less cognitively and physiologically taxing than other emotion regulation strategies that occur after or during an emotional experience.

Research on sleep and emotion regulation to date has solely focused on regulatory strategies that involve how people regulate or control the experience or expression of their negative emotional responses after an emotion-eliciting situation has been encountered. To our knowledge, research has yet to examine associations between sleep patterns and the selection of emotional situations. Prioritizing some activities over others may contribute to the selection of situations that meet immediate needs (e.g., avoiding social interactions because of reduced energy or low motivation). However, this may ultimately serve to undermine emotional experiences that may be beneficial for overall mental health in both the short or long term (e.g., social interactions may produce positive affect and build relationships over time).

Associations between sleep and situation selection may be especially important to understand in adolescence, as teens are developing more autonomy over their daily activities and are faced with more independent decision-making. In addition, adolescents in particular are at risk for problematic sleep patterns, making this a critical age period to examine sleep-emotion relationships. Biologically, adolescence is a period marked by delays in the timing of their melatonin onset, often resulting in later bedtimes (Crowley et al., 2018). When combined with other societal and psychological changes (e.g., greater academic/employment demands, increased autonomy over sleep schedules, early school start times), teens often obtain inadequate amounts of sleep. In addition to high rates of suboptimal sleep duration for 
adolescents, variability in sleep duration and timing peaks during this age period (Dahl and Lewin, 2002; Thorleifsdottir et al., 2002) and many teens attempt to compensate for chronic sleep restriction through extended weekend sleep (social jetlag; Gradisar et al., 2011). Importantly, this increased variability in sleep patterns during the adolescent period has been associated with altered brain development (Telzer et al., 2015) along with increased stress and psychopathology symptoms (Becker et al., 2017).

Additionally, gender differences have been noted regarding how adolescents respond to emotional situations. Females have shown greater tendency to seek social support, but also engage in increased rumination and greater emotional expression compared to males (Zimmermann and Iwanski, 2014; Eschenbeck et al., 2007). Conversely, males report greater suppression, avoidance, and passivity when responding to their emotions (Zimmermann and Iwanski, 2014; Eschenbeck et al., 2007). Less is known about potential gender differences in situation selection as a specific emotion regulation strategy. Research to date has yielded mixed findings regarding gender differences in adolescent sleep. While several studies report no differences in overall sleep duration (e.g., Ohayon et al., 2000; Laberge et al., 2008), others report shortened sleep duration for females (Lee et al., 1999). Data regarding sleep timing and variability is similarly conflicted, with studies suggesting later sleep and rise times for males (Lee et al., 1999; Ohayon et al., 2000) and greater social jetlag in females (Mateo et al., 2012; Lee et al., 1999). Investigation into the gender differences in associations between sleep and emotional responding is limited. However, poorer sleep quality has been associated with greater increases in next day negative affect for girls compared to boys (van Zundert et al., 2015), and sleep deprivation has resulted in greater depressed and anxious mood in female but not male adolescents (Short and Louca, 2015), suggesting that the impact of disrupted sleep may be greater in female adolescents.

The current study examined associations between sleep patterns and situation selection among healthy adolescents. It was expected that more problematic sleep patterns, as assessed 
via actigraphy, would be associated with less avoidance of negative emotional situations, and less selection of positive emotional situations. In particular, we focused on sleep patterns previously associated with emotional symptoms in youth, including sleep duration (Reddy et al., 2017), sleep onset latency (Reynolds and Alfano, 2016; Silk et al., 2007), later sleep timing (Asarnow et al., 2014), and intraindividual variability in sleep duration and sleep timing (Becker et al., 2017). We also explored how associations might differ by gender. We hypothesized that associations between sleep patterns and situation selection would be strongest for girls, in line with previous studies in adolescents that have found that gender moderates relationships between emotional experiences and sleep (Short and Louca, 2015; van Zundert et al., 2015).

\section{Methods}

Participants included 54 healthy adolescents between the ages of $13-17(M=15.19, S D$ $=1.23)$. Adolescents were approximately half female $(61.1 \%)$ and primarily white $(59.6 \% ; 23.1 \%$ Asian, 9.6\% African-American/black, 1.9\% Native American, 5.8\% multiracial; 29.6\% Hispanic/Latino). Most participants came from families with an annual household income greater than $\$ 100 \mathrm{k}$ (59.3\%; $7.4 \%$ between $\$ 80-100 \mathrm{k}, 18.5 \%$ between $\$ 60-80 \mathrm{k}, 9.3 \%$ between $\$ 40-60 \mathrm{k}$, and $5.6 \%$ between $\$ 20-40 k$ ). Participants' caregivers were generally well educated, with $70.4 \%$ of mothers and $59.2 \%$ of fathers with a college or advanced degree $(1.9 \%$ of mothers and $3.7 \%$ of fathers had not completed high school; $7.4 \%$ of mothers and $9.3 \%$ of fathers completed high school; and $20.4 \%$ of mothers and $27.8 \%$ of fathers completed some college). Adolescents were ineligible if they or their primary caregiver did not speak fluent English, were currently receiving treatment for any psychological or sleep problem, had any medical conditions or were taking medication that could impact their sleep or mood, or had been diagnosed with or were suspected to have a sleep disorder. The original study included a total of 58 adolescents, but two participants dropped out of the study prior to completion, and two did not complete 5 nights of actigraphy and were removed from analyses, resulting in the final sample of 54 .

\section{Procedure}


Ethical approval was obtained from the University of Houston Institutional Review Board. Participants were recruited from a large metropolitan area in the United States through local schools and community advertisements. Interested families completed a brief phone interview to assess initial eligibility criteria, which included questions regarding the presence of any psychiatric or sleep disturbances (see Supplemental File 1 for a flow diagram of the total number of participants screened and reasons for exclusion). If eligible, they were scheduled for two in-person assessments approximately 1 week apart. During the first assessment, the adolescent and a legal guardian provided informed assent and consent. Caregivers reported on demographic characteristics and on their child's symptoms, and the adolescent provided reports on their trait situation selection and emotion regulation. Caregivers and adolescents also completed a semi-structured sleep interview together to assess the child's sleep and rule out the presence of any sleep disorders based on International Classification of Sleep Disorders (ICSD-3) criteria. All interviews were reviewed with a Ph.D. level psychologist with training in the diagnosis of sleep disorders.

Following this first assessment, adolescents completed at least one week of actigraphy monitoring and online nightly diaries where they reported on emotion regulation strategies that they engaged in throughout the day (range 7-11 days, median $=8$ days; $n_{\text {reports }}=420,5.62 \%$ missing). Participants returned to the lab following the sleep monitoring period to complete a lab-based situation selection task. The majority of adolescents participated during a school break period (i.e., spring break or summer, $n=35$ ). Participants were compensated with a $\$ 50$ gift card if they completed all portions of the study.

\section{Assessments}

Trait situation selection. Self-reported trait situation selection tendencies were assessed using the Use of Situation Selection questionnaire (Webb et al., 2018), which has been previously validated in two community samples of adults. Participants indicated the extent to which they selected into particular emotional situations on a 5-point scale from 1 (not at all 
like me) to 5 (very much like me). A total of four items assessed selection into positive situations $(\alpha=.85$; i.e., "I select activities that help me to feel good," "If a situation makes me feel good, then I try to stick around," "I gravitate towards people, situations, and activities that put me in a good mood," and "I keep doing something if it seems to be improving my mood."), and two items assessed avoidance of negative situations ( $\alpha=.60$; i.e., "I shy away from situations that might upset me," and "I steer clear of people who put me in a bad mood."). A principal components analysis indicated a 2-factor solution, with a factor representing the selection of positive situations, and a factor representing the avoidance of negative situations (see Supplemental File 2). Higher scores on each subscale indicated either greater selection into positive situations or greater avoidance of negative situations.

Daily situation selection. Every evening during the nightly diaries, participants reported on two items to assess their situation selection throughout the day. Specifically, adolescents were asked to indicate whether or not they selected into positive situations ("Did you purposely or intentionally do anything today to make yourself feel good or happy? For example, talking to a friend or listening to a favorite song?") and if they avoided any negative situations ("Did you purposely or intentionally avoid anything today that you thought might make you feel bad? For example, avoided talking to someone you are mad at or don't like, or decided not to watch a sad or scary movie"). They also provided brief open-ended responses about their situation selection. Two proportion scores were created (number of days the participant indicated they engaged in situation selection / total number of days they provided reports), so that higher scores indicated more selection of positive or more avoidance of negative situations.

Lab-based situation selection. Participants completed a situation selection task adapted from prior research (Livingstone and Isaacowitz, 2015). Participants were provided with a list of 10 positive and 10 negative short video clips (1:13-2:36 minutes in length). Each clip in the list contained a 2-4 word description of the video, followed by the emotion that the video was expected to elicit (e.g., "Seal catches a ride - Happy"). Prior to the start of the task, a research 
assistant informed the adolescent that they could choose any videos in whatever order they wanted, could watch each video as long as they would like, and could stop and start a new video at any time. The research assistant then left the room for 10 minutes, and viewing patterns were recorded using screen capture software. Two total scores were created based on the total number of positive videos and the total number of negative videos that were chosen during the 10-minute period.

Actigraphy. Sleep data was collected using an Actiwatch Spectrum Plus (Philips Respironics). Data was downloaded and processed in one minute epochs using the standard medium sensitivity threshold. During the actigraphy monitoring period, participants also completed a sleep diary prior to bedtime and just after waking, and pushed an event marking button each night when they were first trying to fall asleep. The Philips Actiwatch Spectrum Plus also collects spectrum light data. A combination of sleep diary reports, event markers, and light data was used to identify sleep-wake patterns and artifacts in the data. All participants had at least 5 nights of valid actigraphy data. The following sleep variables were extracted and averaged across the actigraphy monitoring period: total sleep time (TST; total number of minutes slept), sleep onset latency (SOL; minutes taken to fall asleep), sleep onset time (SOT; clock time the participant fell asleep), and midsleep point (MSP; clock time between SOT and when the participant woke in the morning). Using methods from prior research (e.g., Becker et al., 2017), intraindividual variability in TST (IIV TST), SOT (IIV SOT), and MSP (IIV MSP) were calculated using the coefficient of variation $\left[(\sigma / \mu)^{*} 100\right]$.

Depressive symptoms. Adolescent depressive symptoms were assessed using parentreported scores on the Diagnostic and Statistical Manual of Mental Disorders oriented depressive symptoms subscale of the Child Behavior Checklist (Achenbach \& Rescorla, 2001). Parents responded to statements to indicate how well they described their child now or in the last 6 months on a scale from 0 (not true of their child) to 2 (very true or often true of their child). For the current study, we removed four items from this subscale that pertained to sleep. The 
remaining 9 items were averaged to create a total depressive symptoms subscale $(\alpha=.65)$, with higher scores indicating greater depressive symptoms.

Emotion Dysregulation. Participants reported on their overall emotion dysregulation using the 36-item Difficulties with Emotion Regulation Scale (DERS; Gratz \& Roemer, 2004). Participants reported on how often they experienced a range of difficulties that impact emotion regulation after the occurrence of an emotional situation, such as a lack of emotional clarity and emotional awareness, non-acceptance of emotional responses, limited access to emotion regulation strategies, and difficulties with impulse control and goal-directed behavior. Participants reported on these difficulties on a 5-point scale from 1 (almost never) to 5 (almost always). Items were totaled to create an overall emotion dysregulation score $(\alpha=.93)$, with higher scores indicated greater emotion dysregulation.

\section{Analysis Plan}

To assess whether sleep patterns were associated with situation selection, we ran a series of regression analyses, which included the following as dependent variables: trait and daily reports of approaching positive and avoiding negative situations, and the number of positive and negative videos watched during the lab-based situation selection task. Independent variables across the models included TST, SOL, SOT, MSP, IIV TST, IIV SOT, and IIV MSP. To avoid issues with multicollinearity we ran separate models for each sleep predictor. Age, gender, school break status, depressive symptoms, and general emotion dysregulation were included as covariates in all models. Additionally, models including IIV TST, IIV SOT, or IIV MSP also included the average value of the corresponding sleep variable as a covariate (e.g., models including IIV TST included average TST as a covariate). All regression analyses were conducted using SPSS 25, and moderation analyses were conducted using the PROCESS 3.1 with 5,000 bootstrapped samples (Hayes, 2017).

\section{Results}

\section{Preliminary Analyses}


The majority of participants $(90.7 \%)$ obtained less than the $8-10$ hours of sleep recommended by the American Academy of Pediatrics (Paruthi et al., 2016; $M=6.96$ hours, SD $=47.59$ minutes, range $=5.00-8.55$ hours). Teens who participated during the school year obtained less sleep than those who participated during summer/holiday breaks $\left(\mathrm{M}_{\text {school }}=393.12\right.$ minutes, $\mathrm{SD}_{\text {school }}=47.41 ; \mathrm{M}_{\text {break }}=429.84$ minutes, $\left.\mathrm{SD}_{\text {break }}=44.11, t(50)=-2.75, p=.008\right)$, and had a significantly earlier MSP $\left(M_{\text {school }}=3: 31: 22 \mathrm{AM}, \mathrm{SD}_{\text {school }}=00: 57: 00, \mathrm{M}_{\text {break }}=4: 41: 13 \mathrm{AM}\right.$, $\left.\mathrm{SD}_{\text {break }}=1: 29: 59, t(46.28)=-3.40, p=.001\right)$. Girls had greater IIV TST $\left(\mathrm{M}_{\text {girls }}=40.44\right.$ minutes, $\mathrm{SD}_{\text {girls }}=4.83, \mathrm{M}_{\text {boys }}=37.29$ minutes, $\left.\mathrm{SD}_{\text {boys }}=2.74, t(51.50)=-3.05, p=.004\right)$.

Bivariate correlations and descriptive statistics among all variables of interest and covariates are displayed in Table 1. Participants who reported greater emotion dysregulation and who participated during a school break watched less positive videos. Older adolescents reported greater trait approach of positive and avoidance of negative situations, and had a significantly later SOT. Greater general emotion dysregulation was associated with greater depressive symptoms, less avoidance of negative situations based on trait reports, but more avoidance based on daily reports. Greater emotion dysregulation and depressive symptoms were also associated with a later SOT and a later MSP. Additionally, bivariate correlations indicated the total number of videos watched during the lab task was not significantly related to any sleep variable of interest (all p's > .11).

[insert Table 1 about here]

\section{Sleep and Situation Selection}

Regression analyses controlling for age, gender, school break status, depressive symptoms, and emotion dysregulation were conducted (see Tables 2 and 3). Results indicated that greater variability in sleep timing was associated with less avoidance of negative situations. Specifically, IIV SOT was associated with less trait avoidance of negative situations, even when controlling for average SOT. Similarly, greater IIV MSP was associated with less trait avoidance of negative situations and marginally associated with greater selection of negative videos during 
the lab task, even when controlling for average MSP. Additionally, longer SOL was associated with greater avoidance of negative situations based on trait reports, and marginally greater avoidance of negative situations based on the daily reports.

\section{[insert Tables 2 and 3 about here]}

Gender. A series of moderation models were also examined in PROCESS 3.1 (Hayes, 2017). All interaction terms are presented in Tables 4 and 5. All models included age, school break status, depressive symptoms, and emotion dysregulation as covariates. Finally, gender and the interaction term between gender and each sleep variable was also included.

Overall, results from these moderation models indicated that greater variability in sleep timing was associated with reports of approaching positive situations less on the trait and daily reports, but only for boys. Specifically, there was a main effect of IIV SOT on trait reports of approaching positive situations, whereby greater variability in SOT was associated with less approach of positive situations $(\mathrm{B}=-.47, \mathrm{SE}=.20, \mathrm{p}=.027,95 \% \mathrm{Cl}:-.88,-.06)$. However, this was qualified by a significant interaction with gender and IIV SOT (Figure 1). Simple slope analyses indicated that boys with greater variability in their SOT reported that they approached positive situations less than boys who experienced less variability in their sleep timing $(B=-.22$, $\mathrm{SE}=.10, \mathrm{p}=.027,95 \% \mathrm{Cl}:-.42,-.03)$, however this association was not significant for girls $(\mathrm{B}=$ $.02, \mathrm{SE}=.06, \mathrm{p}=.73,95 \% \mathrm{Cl}:-.10, .14)$. There was also a main effect of IIV SOT when predicting daily reports of approaching positive situations $(\mathrm{B}=-.23, \mathrm{SE}=.09, \mathrm{p}=.013,95 \% \mathrm{Cl}$ : $-.41,-.05)$. Greater variability in SOT was associated with less approach of positive situations. However, this main effect was qualified by an interaction with gender (Figure 2). Simple slopes indicated that IIV SOT was associated with approaching less positive situations for boys $(\mathrm{B}=$ $.10, \mathrm{SE}=.04, p=.03,95 \% \mathrm{Cl}:-.19,-.01)$ but not for girls $(\mathrm{B}=.03, \mathrm{SD}=.03, \mathrm{p}=.17,95 \% \mathrm{Cl}$ : $.02, .09)$. No significant interactions emerged for avoidance of negative situations on both the trait and daily reports.

[insert Figures 1 and 2 about here] 
In addition, results also indicated that greater sleep variability was associated with choosing fewer positive movies in the lab task, but only for boys. Specifically, a main effect emerged for IIV MSP, where greater IIV MSP was associated with choosing fewer positive videos during the task $(\mathrm{B}=-.18, \mathrm{SE}=.06, \mathrm{p}=.005,95 \% \mathrm{Cl}:-.30,-.06)$, but this was qualified by an interaction with gender (Figure 3). Simple slope analyses indicated that greater IIV MSP was associated with less selection of positive videos for boys $(B=-.10, S E=.03, p=.007,95 \% \mathrm{Cl}$ : $.13,-.03)$, but not for girls $(\mathrm{B}=.02, \mathrm{SE}=.03, \mathrm{p}=.40,95 \% \mathrm{Cl}:-.03, .07)$. There was also a main effect of IIV TST on positive videos chosen during the lab-based task $(B=-.74$, SE $=.27, p=$ $.008,95 \% \mathrm{Cl}:-1.27,-.20)$, where greater variability in sleep duration was associated with less selection of positive videos. This main effect was further qualified by a significant interaction (Figure 4), where boys with greater IIV TST selected less positive videos during the lab-based task $(\mathrm{B}=-.34, \mathrm{SE}=.13, \mathrm{p}=.01,95 \% \mathrm{Cl}:-.61,-.08)$, whereas IIV TST was not significant for girls $(B=.05$, SE $=.06, p=.38,95 \% \mathrm{Cl}:-.06, .16)$.

\section{[insert Figures 3 and 4 about here]}

Similarly, there was a marginal effect of IIV TST on negative videos, where greater IIV TST was associated with marginally more selection of negative videos $(B=.58, S E=.33, p=$ $.08,95 \% \mathrm{Cl}:-.08,1.24)$, and this was qualified by a marginal interaction. IIV TST was unrelated to selection of negative videos for girls $(B=-.02$, SE $=.07, p=.82,95 \% \mathrm{Cl}:-.16, .12)$, but boys with greater IIV TST selected marginally more negative videos during the task $(\mathrm{B}=.28$, SE $=$ $.16, p=.087,95 \% \mathrm{Cl}:-.04, .61)$. There was also a main effect of TST, where greater TST was associated with greater selection of positive movies $(B=.04, S E=.02, p=.046,95 \% \mathrm{Cl}: .001$, .07). This main effect was qualified by a marginal interaction with gender, although this interaction was only marginally significant $(B=-.02, S E=.01, p=.057,95 \% \mathrm{Cl}:-.04, .001)$. A longer average TST was associated with greater selection of positive movies for boys $(B=.002$, $\mathrm{SE}=.01, \mathrm{p}=.049,95 \% \mathrm{Cl}: .0001, .04)$ but not for girls $(\mathrm{B}=-.002, \mathrm{SE}=.005, \mathrm{p}=.73,95 \% \mathrm{Cl}$ : $.01, .008)$. 
[insert Tables 4 and 5 about here]

\section{Discussion}

Overall, sleep patterns among healthy adolescents were associated with the selection of

emotional situations, with some convergence observed across questionnaires, daily reports, and

a lab-based task. Greater variability in sleep timing was associated with less avoidance of negative situations, whereas a longer sleep onset latency was associated with more avoidance of negative situations. Greater variability in sleep timing and sleep duration were also associated with reduced approach of positive situations, but only for boys. While prior research to our knowledge has yet to examine situation selection in relation to sleep, the use of objective sleep measures and replication across multiple assessments of situation selection strengthen the evidence for the relationship between sleep and this previously unexplored emotion regulation strategy. These associations remained even when controlling for mood symptoms and general emotion dysregulation, providing support that situation selection is uniquely associated with adolescent sleep patterns, over and above other previously explored indicators of emotional health.

The significant association between greater variability in sleep timing and less avoidance of negative emotional situations converges with other findings suggesting that sleep consistency is an important index of healthy sleep (Becker et al., 2017). For example, previous research suggests that children with greater sleep timing variability were more likely to initiate negative interactions at school (Vaughn et al., 2015). We also found that increased sleep onset latency was associated with more avoidance of negative situations in our sample. These avoidant behaviors may circumvent immediate negative feelings (e.g., through avoiding upsetting conversations or difficult tasks), but may paradoxically perpetuate negative affect due to a buildup of avoided responsibilities. This supports studies in youth suggesting that difficulties surrounding bedtime (i.e., sleep initiation) may be associated with later internalizing symptoms 
(Reynolds and Alfano, 2016), and provides a possible mechanism that may underlie these problematic developmental trajectories.

The current study also found associations between sleep variability and the selection of positive emotional situations, corresponding with previous findings that children with more variability in their sleep patterns experience more withdrawn behaviors (Yokomaku et al., 2008), and poor sleep in adults alters motivation and reward processing (Gujar et al., 2011). However, in the current study, these associations were only significant for boys. Specifically, boys with more variability in their sleep patterns were less likely to approach positive situations. While these gender-based differences were consistent across multiple methods, they were unexpected due to prior research suggesting girls are more susceptible to the effects of sleep disturbances (Short and Luca, 2015; van Zundert et al., 2015). However, prior studies have focused on reactivity to emotional situations or experienced affect, and not selection of emotional situations specifically. Further, while post-pubertal girls experience higher rates of depression (Thapar et al., 2012), depressed adolescent boys experience more symptoms of anhedonia (Bennett et al., 2005). The tendency for boys with greater variability in their sleep patterns to seek out fewer positive experiences may be one mechanism by which these hedonic deficits occur.

It is also possible that these identified gender differences may be due to differential stress responses. Girls may be more likely to engage in "tend-and-befriend" as opposed to "fight-or-flight" behaviors in times of high stress, which may lead them to seek out more social interactions (Taylor et al., 2000). Additionally, girls are encouraged to display more happiness in order to minimize interpersonal tension and to prioritize relationship-oriented goals even in difficult situations (Brody, 1999; Brody and Hall, 2008). Post-hoc exploration of the qualitative responses in the daily reports suggested many of the positive situations selected were social. As a result, during times of high stress (when variability in sleep patterns may be common) girls may engage in positive social interactions more than boys. This tendency to maintain social- 
relational goals may have masked the effect of girls' sleep on the approach of positive situations. These differences in how girls and boys process and approach positively-valenced situations would also explain why gender differences emerged for positive and not negative situations.

It is notable that very few findings emerged for average sleep duration despite reliable associations between sleep duration and emotional outcomes in prior research (Palmer and Alfano, 2017). Despite the fact that our sample was healthy and the presence or suspected presence of a sleep disorder was an exclusionary criterion for participation, the majority of our participants were sleep restricted across the actigraphy period, which may have limited our ability to detect effects with sleep duration alone. This is in line with data suggesting that the majority of adolescents are chronically sleep deprived (Owens et al., 2014). However, our sample did have higher variability of sleep duration (between 2-3 hours) compared to averages reported in other adolescent samples (approximately 1.5 hours; Becker et al., 2017). The relative diversity of the current sample may have contributed to this increased variability as nonWhite youth have been found to show greater variability in sleep duration when compared to White youth even after controlling for parental education, income, and neighborhood factors (Becker et al., 2017).

While the current study included an objective sleep assessment and multiple methods to assess situation selection, this research is not without limitations. The small sample size underlies the importance of replicating these findings in future research. Additionally, all analyses were correlational in nature and the directionality of the current findings are unknown. While it is plausible that sleep patterns may predict situation selection, it is also possible that teens who chose poorer emotional situations are also more likely to experience problematic sleep patterns. Future research should examine these processes using experimental designs or should examine daily within-person changes to investigate how changes in sleep patterns from one night to the next may predict engagement in situation selection the following day. Future 
studies could also investigate how these emotional strategies may predict subsequent fluctuations in sleep. Additionally, while we found associations among sleep patterns and overall tendencies to select into positive or negative situations, determining whether situation selection is adaptive depends on the context in which it occurs (Aldao, 2013), and future research should consider how teens are flexible in their emotion regulation strategy use to meet current situational demands. Future studies should also examine how sleep may be associated with situation selection within contexts that may be more salient to boys or girls (e.g., risk-taking behavior, interpersonal interactions). Exploration of individual differences (other than gender) is also needed. For example, adolescents experience greater anhedonia than adults (Spear, 2000), so examining how differences in the selection of positive situations may differ by age is an important future direction. Further, a greater understanding of the mechanisms underlying sleep IIV-emotion associations is needed (e.g., variability in sleep timing may result in increased circadian misalignment and/or altered cognitive/physiological arousal).

Emotion research has highlighted situation selection strategies as one of the most effective forms of regulation (Duckworth et al., 2016; Gross, 1998); thus, these findings deepen our understanding of sleep-emotion relationships and suggest that deficits in mood may begin even before emotional situations are encountered. Adolescents with problematic sleep patterns may expose themselves to situations that are less conducive to experiencing healthy emotions (e.g., more negative and less positive situations), which is problematic when combined with dysregulated responses in response to emotional events as they unfold (Baum et al., 2014). These associations inform developmental models of psychopathology and indicate that situation selection may be one mechanism resulting in sleep-based risk for emotional disorders. 


\section{References}

1. Achenbach, T.M., and Rescorla, L.A. Manual for the ASEBA School-Age Forms \& Profiles. Burlington, VT: University of Vermont, Research Center for Children, Youth, \& Families.

2. Aldao, A. The future of emotion regulation research: Capturing context. Perspect Psychol Sci, 2013, 8: 155-172.

3. Asarnow, L.D., McGlinchey, E., and Harvey, A.G. The effects of bedtime and sleep duration on academic and emotional outcomes in a nationally representative sample of adolescents. J. Adolesc. Health, 2014, 54: 350-356.

4. Baum, K.T., Desai, A., Field, J., Miller, L.E., Rausch, J., and Beebe, D.W. Sleep restriction worsens mood and emotion regulation in adolescence. J. Child Psychol. Psychiatry, 2014, 55: 180-190.

5. Becker, S.P., Sidol, C.A., Van Dyk, T., Epstein, J.N., and Beebe, D.W. Intraindividual variability of sleep/wake patterns in relation to child and adolescent functioning. Sleep Med. Rev., 2017, 34: 94-121.

6. Bennett, D.S., Ambrosini, P.J., Kudes, D., Metz, C., and Rabinovich, H. Gender differences in adolescent depression: Do symptoms differ for boys and girls? J. Affect. Disord., 2005, 89: 35-44.

7. Brody, L.R. Gender, Emotion, and the Family. Harvard University Press, Cambridge, 1999.

8. Brody, L.R., and Hall, J.A. Gender and emotion in context. In: M. Lewis, J.M. HavilandJones, and L.F. Barrett (eds.), Handbook of emotions (pp. 395-408). Guilford Press, New York, 2008.

9. Casey, B. J., Jones, R. M., and Hare, T. A. The adolescent brain. Ann. N. Y. Acad. Sci., 2008, 1124: 111-126.

10. Crowley, S.J., Wolfson, A.R., Tarokh, L., and Carksadon, M.A. An update on adolescent sleep: New evidence informing the perfect storm model. J. Adolesc., 2018, 67: 55-65.

11. Dahl, R. E., and Lewin, D. S. Pathways to adolescent health sleep regulation and behavior. J. Adolesc. Health, 2002, 31: 175-184.

12. Duckworth, A.L., Gendler, T.S., and Gross, J.J. Situational strategies for self-control. Perspect. Psych. Sci., 2016, 11: 35-55.

13. Eschenbeck, H., Kohlmann, C.W., and Lohaus, A. Gender differences in coping strategies in children and adolescents. J. Individ, Dif., 2007, 28: 18-26.

14. Fredriksen, K., Rhodes, J., Reddy, R., and Way, N. Sleepless in Chicago: tracking the effects of adolescent sleep loss during the middle school years. Child Dev., 2004,75: 8495.

15. Gradisar, M., Gardner, G. and Dohnt, H. Recent worldwide sleep patterns and problems during adolescence: A review and meta-analysis of age, region, and sleep. Sleep Med., 2011, 12: 110-118.

16. Gratz, K.L., and Roemer, L. Multidimensional assessment of emotion regulation and dysregulation: Development, factor structure, and initial validation of the Difficulties in Emotion Regulation Scale. J. of Psychpathol. Behav. Assess., 2004, 26: 41-54.

17. Greene, G., Gregory, A.M., Fone, D., and White, J. Childhood sleeping difficulties and depression in adulthood: The 1970 British Cohort Study. J. Sleep Res., 2015, 24: 19-23.

18. Gross, J.J. The emerging field of emotion regulation: An integrative review. Rev. Gen. Psych., 1998, 2: 271-299.

19. Gujar, N., McDonald, S.A., Nishida, M., and Walker, M.P. A role for REM sleep in recalibrating the sensitivity of the human brain to specific emotions. Cereb. Cortex, 2011, 21: 115-123. 
20. Harvey, A.G., Murray, G., Chandler, R.A., and Soehner, A. Sleep disturbance as transdiagnostic: Consideration of neurobiological mechanisms. Clin. Psychol. Rev., 2011, 31: 225-235.

21. Hayes, A.F. Introduction to Mediation, Moderation, and Conditional Process Analysis: A Regression-Based Approach. Guilford Press, New York, 2017 (second edition).

22. Keyes, K.M., Maslowsky, J., Hamilton, A., and Schulenberg, J. The great sleep recession: Changes in sleep duration among US adolescents, 1991-2012. Pediatrics, 2015, 135: 460-468.

23. Laberge L., Petit D., Simard C., Vitaro F., Tremblay RE, and Montplaisir J. Development of sleep patterns in early adolescence. Journal of sleep research. 2001, 10: 59-67.

24. Lee K.A., Mcenany G., and Weekes D. Gender differences in sleep patterns for early adolescents. Journal of adolescent health. 1999, 24:16-20.

25. Livingstone, K.M., and Isaacowitz, D.M. Situation selection and modification for emotion regulation in younger and older adults. Soc. Psychol. Personal. Sci., 2015, 6: 904-910.

26. Mateo M.J., Díaz-Morales, J.F., Barreno, C.E., Prieto, P.D., and Randle,r C. Morningness-eveningness and sleep habits among adolescents: age and gender differences. Psicothema, 2012, 24: 410-5.

27. McGlinchey, E.L., Talbot, L.S., Chang, K., Kaplab, K.A., Dahl, R.E., and Harvey, A.G. The effect of sleep deprivation on vocal expression of emotion in adolescents and adults. Sleep, 2011, 34: 1233-1241.

28. Ohayon, M.M., Roberts, R.E., Zulley, J., Smirne, S., and Priest, R.G. Prevalence and patterns of problematic sleep among older adolescents. J. Am. Acad. Child Adolesc. Psychiatry, 2000, 39:1549-56.

29. Owens, J., and Adolescent Sleep Working Group. Insufficient sleep in adolescents and young adults: An update on causes and consequences. Pediatrics, 2014, 134: e921e932.

30. Palmer, C.A., and Alfano, C.A. Sleep and emotion regulation: An organizing, integrative review. Sleep Med. Rev., 2017, 31: 6-16.

31. Palmer, C.A., Oosterhoff, B., Bower, J.L., Kaplow, J.B., and Alfano, C.A. Associations among adolescent sleep problems, emotion regulation, and affective disorders: Findings from a nationally representative sample. J. Psychiatry Res., 2018, 96: 1-8.

32. Paruthi, S., Brooks, L.J., D'Ambrosio, C. et al. Recommended amount of sleep for pediatric populations: A consensus statement of the American Academy of Sleep Medicine. J. Clin. Sleep Med., 2016, 12: 785-786.

33. Reddy, R., Palmer, C.A., Jackson, C., Farris, S.G., and Alfano, C.A. Impact of sleep restriction versus idealized sleep on emotional experience, reactivity, and regulation in healthy adolescents. J. Sleep Res., 2017, 26: 516-525.

34. Reynolds, K.C., and Alfano, C.A. Childhood bedtime problems predict adolescent internalizing symptoms through emotional reactivity. J. Pediatric Psychol., 2016, 41: 971982.

35. Short, M.A., and Louca, M. Sleep deprivation leads to mood deficits in healthy adolescents. Sleep Med., 2015, 16: 987-993.

36. Silk, J.S., Vanderbilt-Adriance, E., Shaw, D.S. et al. Resilience among children and adolescents at risk for depression: Mediation and moderation across social and neurobiological contexts. Dev. Psychopathol., 2007, 19: 841-865.

37. Spear, L.P. The adolescent brain and age-related behavioral manifestations. Neurosci. Biobehav. Rev., 2000, 24: 417-463.

38. Talbot, L.S., McGlinchey, E.L., Kaplan, K.A., Dahl, R.E., and Harvey, A.G. Sleep deprivation in adolescents and adults: Changes in affect. Emotion, 2010, 10: 831-841. 
39. Taylor, S.E., Cousino Klein, L., Lewis, B.P., Gruenewald, T.L., Gurung, R.A.R., and Updegraff, J.A. (2000). Biobehavioral responses to stress in females: Tend-andbefriend, not fight-or-flight. Psychol. Rev., 2000, 107: 411-429.

40. Telzer, E.H., Goldenberg, D., Fuligni, A.J., Lieberman, M.D., and Galvan, A. Sleep variability in adolescence is associated with altered brain development. Dev. Cogn. Neurosci., 2015, 14: 16-22.

41. Thapar, A., Collishaw, S., Pine, D.S., and Thapar, A.K. Depression in adolescence. Lancet, 2012, 379: 1056-1067.

42. Thorleifsdottir, B., Björnsson, J.K., Benediktsdottir, B., Gislason, T., and Kristbjarnarson, $\mathrm{H}$. Sleep and sleep habits from childhood to young adulthood over a 10-year period. J. Psychosom. Res., 2002, 53: 529-537.

43. van Zundert, R.M., van Roekel, E., Engels, R.C., and Scholte, R.H. Reciprocal associations between adolescents' night-time sleep and daytime affect and the role of gender and depressive symptoms. J. Youth Adolesc., 2015, 44: 556-569.

44. Vaughn, B.E., Elmore-Staton, L., Shin, N., and El-Sheikh, M. Sleep as a support for social competence, peer relations, and cognitive functioning in preschool children. Behav. Sleep Med., 2015, 13: 92-106.

45. Webb, T.L., Lindquist, K.A., Jones, K., Avishai, A., and Sheeran, P. Situation selection is a particularity effective emotion regulation strategy for people who need help regulation their emotions. Cogn. Emot., 2018, 32: 231-248.

46. Yokomaku, A., Misao, K., Omoto, F. et al. A study of the association between sleep habits and problematic behaviors in preschool children. Chronobiol. Int., 2008, 25: 549564.

47. Zimmermann, P., Iwanski, A. Emotion regulation from early adolescence to emerging adulthood and middle adulthood: Age differences, gender differences, and emotionspecific developmental variations. Int. J. Behav. Dev., 2014, 38: 182-94. 
Table 1

Bivariate Correlations among Study Variables and Covariates

\begin{tabular}{|c|c|c|c|c|c|c|c|c|c|c|c|c|c|c|c|c|c|c|}
\hline & 1 & 2 & 3 & 4 & 5 & 6 & 7 & 8 & 9 & 10 & 11 & 12 & 13 & 14 & 15 & 16 & 17 & 18 \\
\hline \multicolumn{19}{|l|}{ 1.Age } \\
\hline 2.Gender & .22 & & & & & & & & & & & & & & & & & \\
\hline 3.Break & $-.28^{*}$ & .04 & & & & & & & & & & & & & & & & \\
\hline 4. DERS & -.01 & .12 & .15 & & & & & & & & & & & & & & & \\
\hline 5. Mood Symptoms & .05 & .08 & .20 & $.41^{* *}$ & & & & & & & & & & & & & & \\
\hline 6. Trait SS - P & $.33^{*}$ & .24 & -.16 & .01 & -.05 & & & & & & & & & & & & & \\
\hline 7.Trait SS - N & $.31^{*}$ & .07 & -.18 & $-.27^{*}$ & -.17 & .22 & & & & & & & & & & & & \\
\hline 8.Daily SS - P & -.19 & .02 & .22 & .10 & .05 & .13 & -.05 & & & & & & & & & & & \\
\hline 9.Daily SS - N & -.02 & .12 & -.13 & $.46^{\star * *}$ & .21 & .03 & .09 & $.29^{*}$ & & & & & & & & & & \\
\hline 10.Lab SS - P & .16 & -.08 & $-.29^{*}$ & $-.28^{*}$ & -.19 & .12 & .22 & -.24 & -.12 & & & & & & & & & \\
\hline 11.Lab SS - N & .14 & .23 & -.13 & .10 & .07 & .01 & -.07 & .04 & .004 & $-.56^{\star * \star}$ & & & & & & & & \\
\hline 12.TST (min) & -.17 & .08 & $.36^{* *}$ & .07 & .04 & -.14 & .01 & -.04 & -.003 & .02 & -.22 & & & & & & & \\
\hline 13.SOL (min) & -.16 & -.08 & .19 & .23 & .26 & .04 & .17 & .15 & $.43^{*}$ & -.23 & .13 & .04 & & & & & & \\
\hline 14.SOT & $.27^{*}$ & .15 & .25 & $.31^{*}$ & $.27^{+}$ & .11 & .02 & .06 & .06 & -.06 & .13 & -.13 & .08 & & & & & \\
\hline 15.MSP & .20 & .16 & $.38^{* *}$ & $.30^{*}$ & $.30^{*}$ & .07 & -.004 & .06 & .05 & -.06 & .07 & .14 & .12 & $.95^{\star \star \star}$ & & & & \\
\hline 16.IIV TST & .22 & $.35^{\star *}$ & -.11 & .16 & .13 & .18 & .06 & .08 & .21 & -.02 & .21 & $-.35^{* *}$ & .07 & $.40^{\star *}$ & $.30^{*}$ & & & \\
\hline 17.IIV SOT & -.03 & .05 & .04 & .06 & -.001 & -.09 & $-.31^{*}$ & .04 & .04 & -.05 & .14 & -.15 & .01 & .25 & .23 & $.47^{* * *}$ & & \\
\hline 18.IIV MSP & -.17 & -.14 & -.15 & -.13 & -.04 & -.09 & $-.28^{*}$ & -.10 & -.24 & -.10 & .17 & -.25 & -.003 & -.24 & $-.30^{*}$ & .16 & $.57^{\star \star \star}$ & \\
\hline Mean & - & - & - & 79.59 & 0.10 & 4.19 & 3.63 & 1.61 & 1.13 & 3.32 & 3.15 & 417.56 & 17.93 & $00: 27$ & $04: 16$ & 39.23 & 4.01 & 24.32 \\
\hline SD & - & - & - & 22.41 & 0.17 & 0.74 & 0.97 & 0.32 & .15 & 1.65 & 1.71 & 47.59 & 18.41 & $01: 27$ & $01: 27$ & 4.40 & 2.11 & 11.47 \\
\hline Minimum & - & - & - & 44.00 & 0.00 & 2.25 & 1.50 & 1.00 & 1.00 & 0.00 & 0.00 & 300.11 & 3.00 & $21: 25$ & $01: 43$ & 30.37 & 0.65 & 6.72 \\
\hline Maximum & - & - & - & 147.00 & 0.78 & 5.00 & 5.00 & 2.00 & 1.67 & 9.00 & 8.00 & 513.00 & 94.47 & $04: 10$ & $07: 47$ & 52.15 & 9.78 & 58.96 \\
\hline
\end{tabular}

Notes. ${ }^{* \star *} p<.001,{ }^{* *} p<.01,{ }^{*} p<.05,+p=.05$. Gender is coded as $1=$ boys and $2=$ girls. Break is coded as $1=$ on school break and $0=$ not on school break.

Trait SS $-\mathrm{P}=$ trait reports of approaching positive situations. Trait $\mathrm{SS}-\mathrm{N}=$ trait reports of avoiding negative situations. Daily $\mathrm{SS}-\mathrm{P}=$ daily reports of

approaching positive situations. Daily $\mathrm{SS}-\mathrm{N}=$ daily reports of avoiding negative situations. Lab $\mathrm{SS}-\mathrm{P}=$ number of positive videos chosen during the situation

selection lab task. Lab SS $-\mathrm{N}=$ number of negative videos chosen during the situation selection lab task. TST = total sleep time. SOL = sleep onset latency. SOT = sleep onset time. $\mathrm{MSP}=$ midsleep point. IIV = intraindividual variability, calculated as the coefficient of variation. 
Table 2

Regression Analyses with Sleep Variables Predicting Situation Selection of Positive Situations on Trait Reports, Daily Questionnaires, and the Lab Task

\begin{tabular}{|c|c|c|c|c|c|c|c|c|c|c|c|c|c|c|c|}
\hline & \multicolumn{5}{|c|}{ Situation Selection Trait - Positive } & \multicolumn{5}{|c|}{ Situation Selection Daily - Positive } & \multicolumn{5}{|c|}{ Situation Selection Lab Task - Positive } \\
\hline & $R^{2}$ & $B$ & SE & Beta & $95 \% \mathrm{Cl}$ & $R^{2}$ & $\mathrm{~B}$ & SE & Beta & $95 \% \mathrm{Cl}$ & $R^{2}$ & $B$ & SE & Beta & $95 \% \mathrm{Cl}$ \\
\hline TST & .15 & -.001 & .002 & -.09 & $-.01, .003$ & .08 & -.001 & .001 & -.14 & $-.003,001$ & .17 & .003 & .004 & .09 & $-.01, .01$ \\
\hline SOL & .14 & .003 & .01 & .10 & $-.01, .02$ & .19 & -.001 & .003 & -.02 & $-.01, .01$ & .18 & -.01 & .02 & -.11 & $-.05, .03$ \\
\hline SOT & .14 & .001 & .001 & .05 & $-.001, .001$ & .07 & .001 & .001 & .07 & $-.001, .001$ & .16 & -.001 & .001 & -.03 & $-.001, .001$ \\
\hline MSP & .14 & .001 & .001 & .04 & $-.001, .001$ & .06 & .001 & .001 & .03 & $-.001, .001$ & .16 & .001 & .001 & .01 & $-.001, .001$ \\
\hline IIV TST & .15 & .003 & .03 & .02 & $-.06, .06$ & .08 & .007 & .01 & .10 & $-.02, .03$ & .17 & -.01 & .06 & -.02 & $-.12, .11$ \\
\hline IIV SOT & .16 & -.04 & .05 & -.12 & $-.15, .06$ & .07 & .001 & .02 & .01 & $-.05, .05$ & 16 & .003 & 10 & .004 & $-.21, .21$ \\
\hline IIV MSP & .15 & -.003 & .01 & -.04 & $-.02, .02$ & .07 & -.003 & .01 & -.09 & $-.01, .01$ & .18 & -.03 & .02 & -.18 & $-.07, .02$ \\
\hline
\end{tabular}

Notes. TST = total sleep time. SOL = sleep onset latency. SOT = sleep onset time. MSP = midsleep point. IIV = intraindividual variability, calculated as the coefficient of variation. All models included age, gender, school break status, emotion dysregulation, and depressive symptoms as covariates. Models with IIV TST also included average TST as a covariate. Models with IIV SOT also included average SOT as a covariate. Models with IIV MSP also included average MSP as a covariate. 
Table 3

Regression Analyses with Sleep Variables Predicting Situation Selection of Negative Situations on Trait Reports, Daily Questionnaires, and the Lab Task

\begin{tabular}{|c|c|c|c|c|c|c|c|c|c|c|c|c|c|c|c|}
\hline & \multicolumn{5}{|c|}{ Situation Selection Trait - Negative } & \multicolumn{5}{|c|}{ Situation Selection Daily - Negative } & \multicolumn{5}{|c|}{ Situation Selection Lab Task - Negative } \\
\hline & $R^{2}$ & $\mathrm{~B}$ & SE & Beta & $95 \% \mathrm{Cl}$ & $R^{2}$ & $\mathrm{~B}$ & SE & Beta & $95 \% \mathrm{Cl}$ & $R^{2}$ & $\mathrm{~B}$ & SE & Beta & $95 \% \mathrm{Cl}$ \\
\hline TST & .17 & .002 & .003 & .10 & $-.004, .01$ & $.26^{*}$ & .001 & .001 & .02 & $-.001, .001$ & .14 & -.01 & .01 & -.19 & $-.02, .004$ \\
\hline SOL & $.40++$ & .02 & .01 & $.42^{*}$ & $.002, .04$ & $.57^{* *}$ & .003 & .001 & $.30++$ & $.001, .005$ & .16 & .01 & .02 & .13 & $-.03, .05$ \\
\hline SOT & .16 & .001 & .001 & .04 & $-.001, .001$ & $.26^{\star}$ & -.001 & .001 & -.04 & $-.001, .001$ & .13 & .001 & .001 & .16 & $-.001, .001$ \\
\hline MSP & .16 & .001 & .001 & .05 & $-.001, .001$ & $.26^{\star}$ & -.001 & .001 & -.03 & $-.001, .001$ & .12 & .001 & .001 & .11 & $-.001, .001$ \\
\hline IIV TST & .17 & .02 & .04 & .08 & $-.06, .09$ & $.27^{*}$ & .01 & .01 & .14 & $-.01, .02$ & .15 & .03 & .07 & .07 & $-.11, .16$ \\
\hline IIV SOT & $.26+$ & -.16 & .06 & $-.34^{*}$ & $-.28,-.03$ & $.26+$ & .003 & .01 & .04 & $-.02, .02$ & .14 & .08 & .12 & .10 & $-.17, .32$ \\
\hline IIV MSP & $.26+$ & -.03 & .01 & $-.33^{*}$ & -.06. -.01 & $.30^{*}$ & -.003 & .002 & -.22 & $-.01, .001$ & .20 & .05 & .02 & $.27+$ & $-.001, .09$ \\
\hline
\end{tabular}

Notes. ${ }^{* *} p<.01,{ }^{*} p<.05,+p=.05,++p<.07 . \mathrm{TST}=$ total sleep time. SOL = sleep onset latency. SOT = sleep onset time. MSP = midsleep point. IIV $=$

intraindividual variability, calculated as the coefficient of variation. All models included age, gender, school break status, emotion dysregulation, and depressive symptoms as covariates. Models with IIV TST also included average TST as a covariate. Models with IIV SOT also included average SOT as a covariate. Models with IIV MSP also included average MSP as a covariate. 
Table 4

Moderation Analyses with Sleep and Gender Predicting Situation Selection of Positive Situations on Trait Reports, Daily Questionnaires, and the Lab Task

\begin{tabular}{|c|c|c|c|c|c|c|c|c|c|c|c|c|}
\hline & \multicolumn{4}{|c|}{ Situation Selection Trait - Positive } & \multicolumn{4}{|c|}{ Situation Selection Daily - Positive } & \multicolumn{4}{|c|}{ Situation Selection Lab Task - Positive } \\
\hline & $R^{2}$ & $\mathrm{~B}$ & SE & $95 \% \mathrm{Cl}$ & $R^{2}$ & $\mathrm{~B}$ & SE & $95 \% \mathrm{Cl}$ & $R^{2}$ & $\mathrm{~B}$ & SE & $95 \% \mathrm{Cl}$ \\
\hline TSTxGender & .15 & -.001 & .01 & $-.01, .01$ & .08 & .001 & .002 & $-.004, .01$ & $.24++$ & $-.02+$ & .01 & $-.04, .001$ \\
\hline SOLxGender & .15 & -.01 & .02 & $-.05, .03$ & .23 & -.01 & .01 & $-.03, .01$ & .19 & -.02 & .05 & $-.13, .08$ \\
\hline SOTxGender & .16 & .001 & .001 & $-.001, .001$ & .08 & .001 & .001 & $-.001, .001$ & .17 & -.001 & .001 & $-.001, .001$ \\
\hline MSPxGender & .15 & .001 & .001 & $-.001, .001$ & .07 & .001 & .001 & $-.001, .001$ & .20 & -.001 & .001 & $-.001, .001$ \\
\hline IIV TSTxGender & 16 & .07 & .08 & $-.09, .22$ & .09 & .02 & .04 & $-.05, .09$ & $.29^{*}$ & $.39^{\star *}$ & .14 & $.11, .68$ \\
\hline IIV SOTxGender & .24 & $.24^{*}$ & .11 & $.01, .47$ & .20 & $.13^{* *}$ & .05 & $.03, .24$ & .18 & .26 & .23 & $-.19, .74$ \\
\hline IIV MSPxGender & .15 & .01 & .02 & $-.03, .06$ & .12 & .01 & .01 & $-.004, .03$ & $.31^{*}$ & $.10^{\star *}$ & .04 & $.02, .17$ \\
\hline
\end{tabular}

Notes. ${ }^{*} p \leq .01,{ }^{*} p<.05,+p=.05,++p<.10 . \mathrm{TST}=$ total sleep time. SOL = sleep onset latency. SOT = sleep onset time. MSP = midsleep point. IIV = intraindividual variability, calculated as the coefficient of variation. All models included age, school break status, emotion dysregulation, and depressive symptoms as covariates. Models with IIV TST also included average TST as a covariate. Models with IIV SOT also included average SOT as a covariate. Models with IIV MSP also included average MSP as a covariate. 
Table 5

Moderation Analyses with Sleep and Gender Predicting Situation Selection of Negative Situations on Trait Reports, Daily Questionnaires, and the Lab Task

\begin{tabular}{|c|c|c|c|c|c|c|c|c|c|c|c|c|}
\hline & \multicolumn{4}{|c|}{ Situation Selection Trait - Negative } & \multicolumn{4}{|c|}{ Situation Selection Daily - Negative } & \multicolumn{4}{|c|}{ Situation Selection Lab Task - Negative } \\
\hline & $R^{2}$ & $\mathrm{~B}$ & $\mathrm{SE}$ & $95 \% \mathrm{Cl}$ & $R^{2}$ & $\mathrm{~B}$ & SE & $95 \% \mathrm{Cl}$ & $R^{2}$ & $\mathrm{~B}$ & $\mathrm{SE}$ & $95 \% \mathrm{Cl}$ \\
\hline TSTxGender & .19 & .01 & .01 & $-.01, .02$ & $.52^{*}$ & -.001 & .001 & $-.003, .001$ & .18 & .02 & .01 & $-.01, .04$ \\
\hline SOLxGender & .41 & .01 & .02 & $-.04, .06$ & $.58^{\star *}$ & .003 & .004 & $-.004, .01$ & .17 & -.03 & .05 & -.14 .09 \\
\hline SOTxGender & .18 & -.001 & .001 & $-.001, .001$ & $.26+$ & .001 & .001 & $-.001, .001$ & .13 & .001 & .001 & $-.001, .001$ \\
\hline MSPxGender & .17 & .001 & .001 & $-.001, .001$ & $.26+$ & .001 & .001 & $-.001, .001$ & .13 & .001 & .001 & $-.001, .001$ \\
\hline IIV TSTxGender & .17 & -.03 & .10 & $-.24, .17$ & $.27++$ & -.004 & .04 & $-.03, .03$ & .20 & $-.30++$ & .17 & $-.65, .05$ \\
\hline IIV SOTxGender & $.28^{*}$ & .18 & .14 & $-.11, .47$ & .26 & .01 & .02 & $-.04, .05$ & .16 & -.29 & .27 & $-.83, .26$ \\
\hline IIV MSPxGender & $.27++$ & .03 & .02 & $-.02, .08$ & $.30^{*}$ & .001 & .003 & $-.01, .01$ & .22 & -.05 & .05 & $-.15, .04$ \\
\hline
\end{tabular}

Notes. ${ }^{* \star} p<.01,{ }^{*} p<.05,+p=.05,++p<.10$. TST = total sleep time. SOL = sleep onset latency. SOT = sleep onset time. MSP = midsleep point. IIV =

intraindividual variability, calculated as the coefficient of variation. All models included age, school break status, emotion dysregulation, and depressive symptoms as covariates. Models with IIV TST also included average TST as a covariate. Models with IIV SOT also included average SOT as a covariate. Models with IIV MSP also included average MSP as a covariate. 


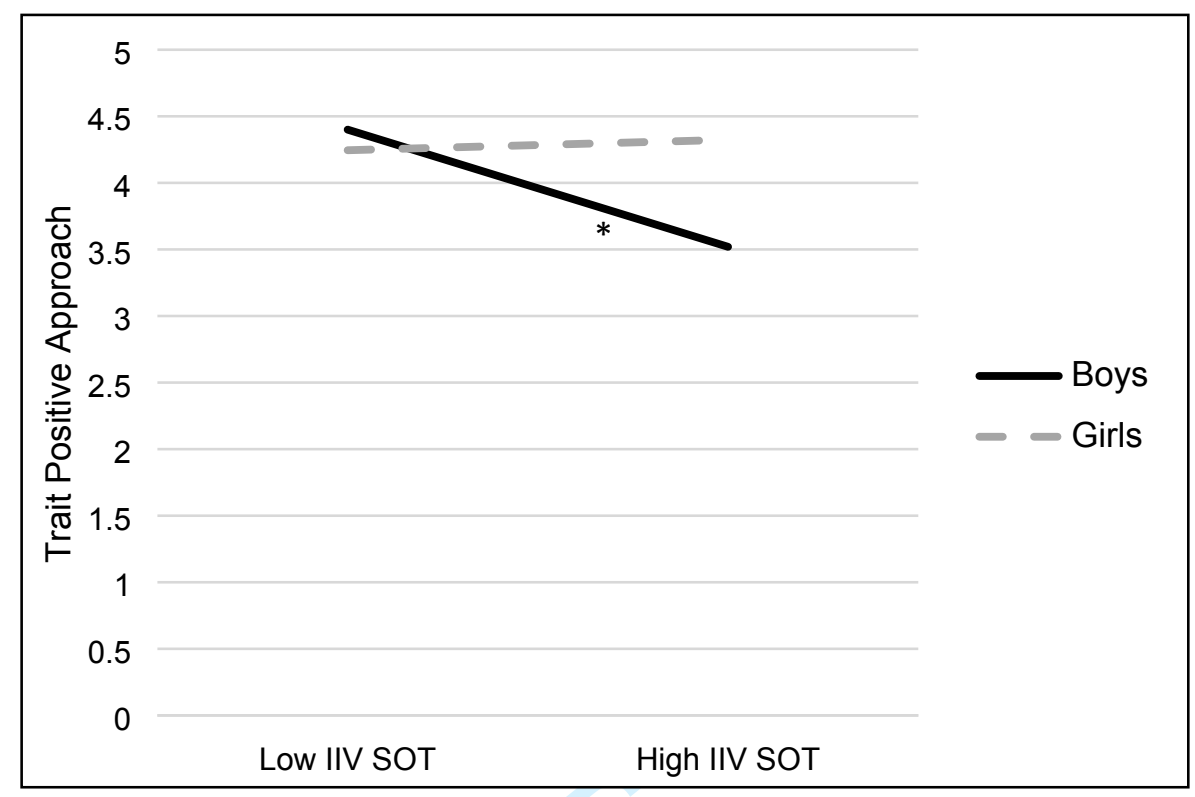

Figure 1. Interaction between gender and intraindividual variability of sleep onset time predicting trait reports of selecting into positive situations.

Notes: IIV SOT = intraindividual variability in sleep onset timing. Higher values on the y-axis indicate greater approach of positive situations. 


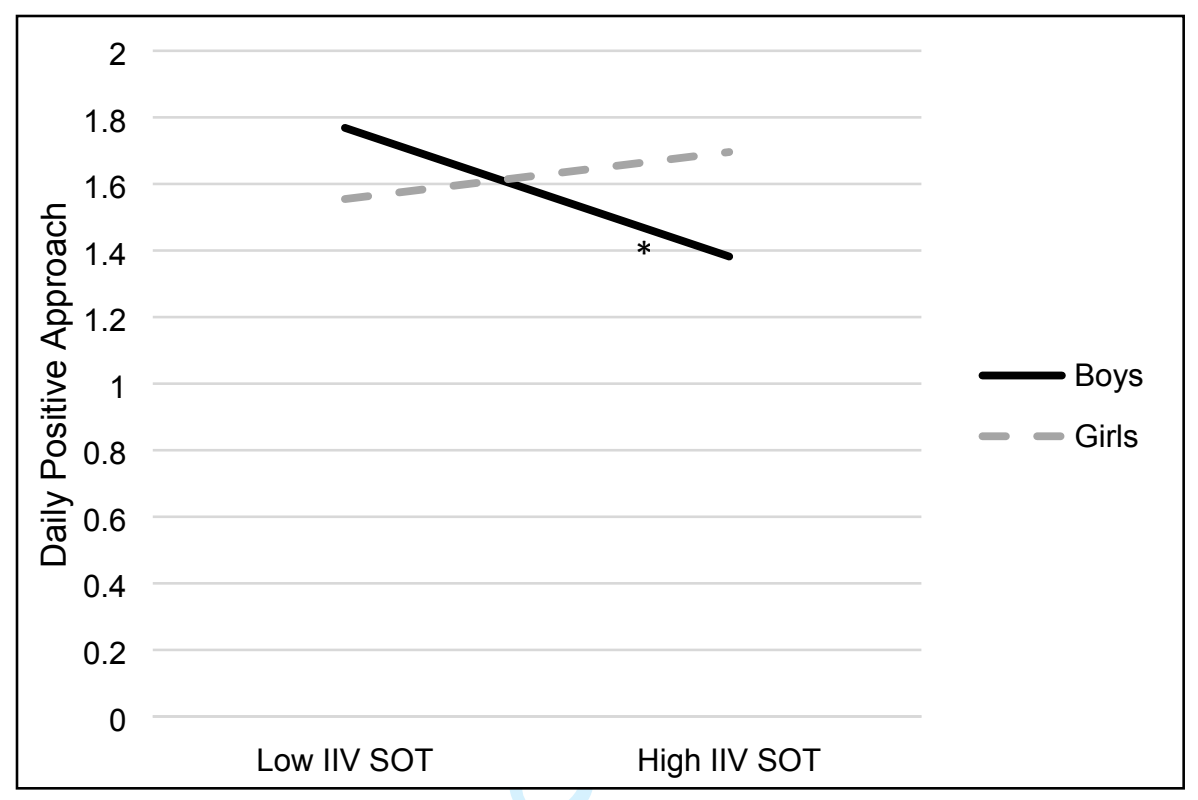

Figure 2. Interaction between gender and intraindividual variability in sleep onset time predicting daily reports of selecting into positive situations.

Notes: IIV SOT = intraindividual variability in sleep onset timing. Higher values on the y-axis indicate greater approach of positive situations. 


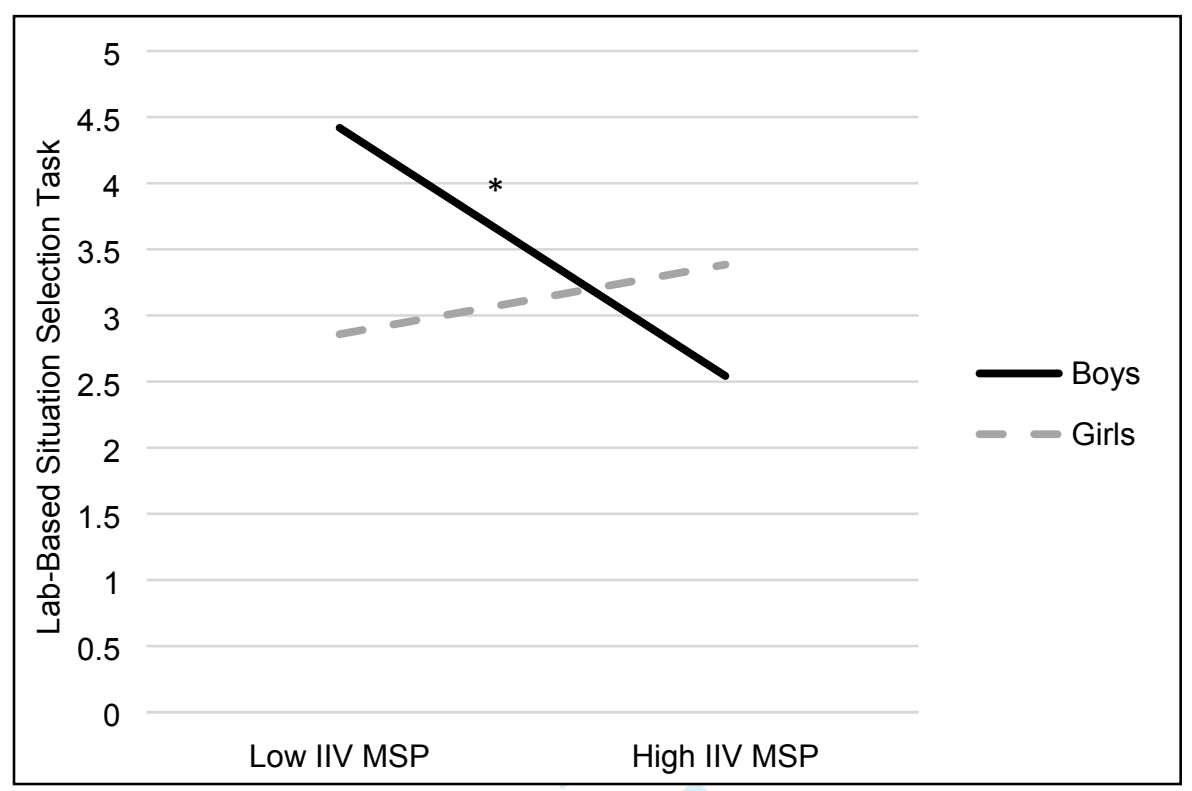

Figure 3. Interaction between gender and intraindividual variability in sleep timing predicting selection of positive videos during the lab task.

Notes: IIV MSP = intraindividual variability in midsleep point. Higher values on the y-axis indicate greater selection of positive (compared to negative) videos during the lab based task. 


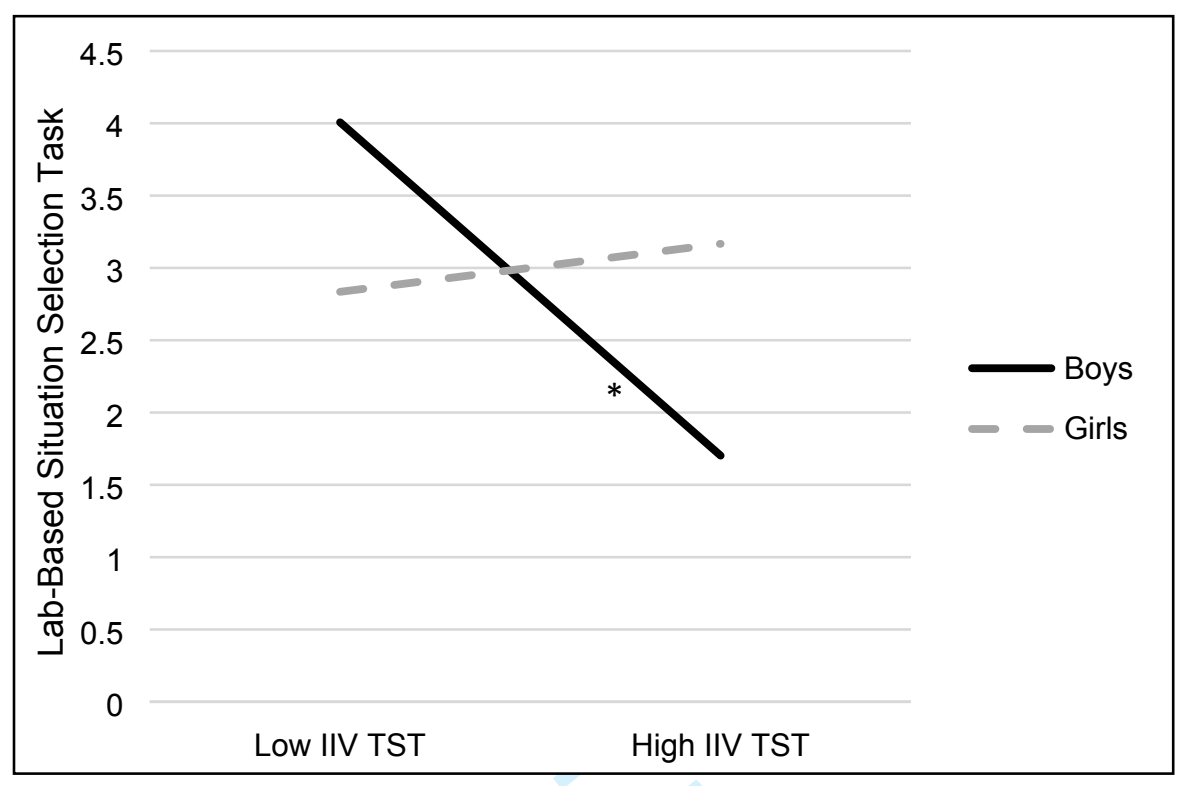

Figure 4. Interaction between gender and intraindividual variability in sleep duration predicting selection of positive videos during the lab task.

Notes: IIV TST = intraindividual variability in total sleep time. Higher values on the y-axis indicate greater selection of positive (compared to negative) videos during the lab based task. 


\section{Supplemental File 1:}

Participant Inclusion Flow Chart

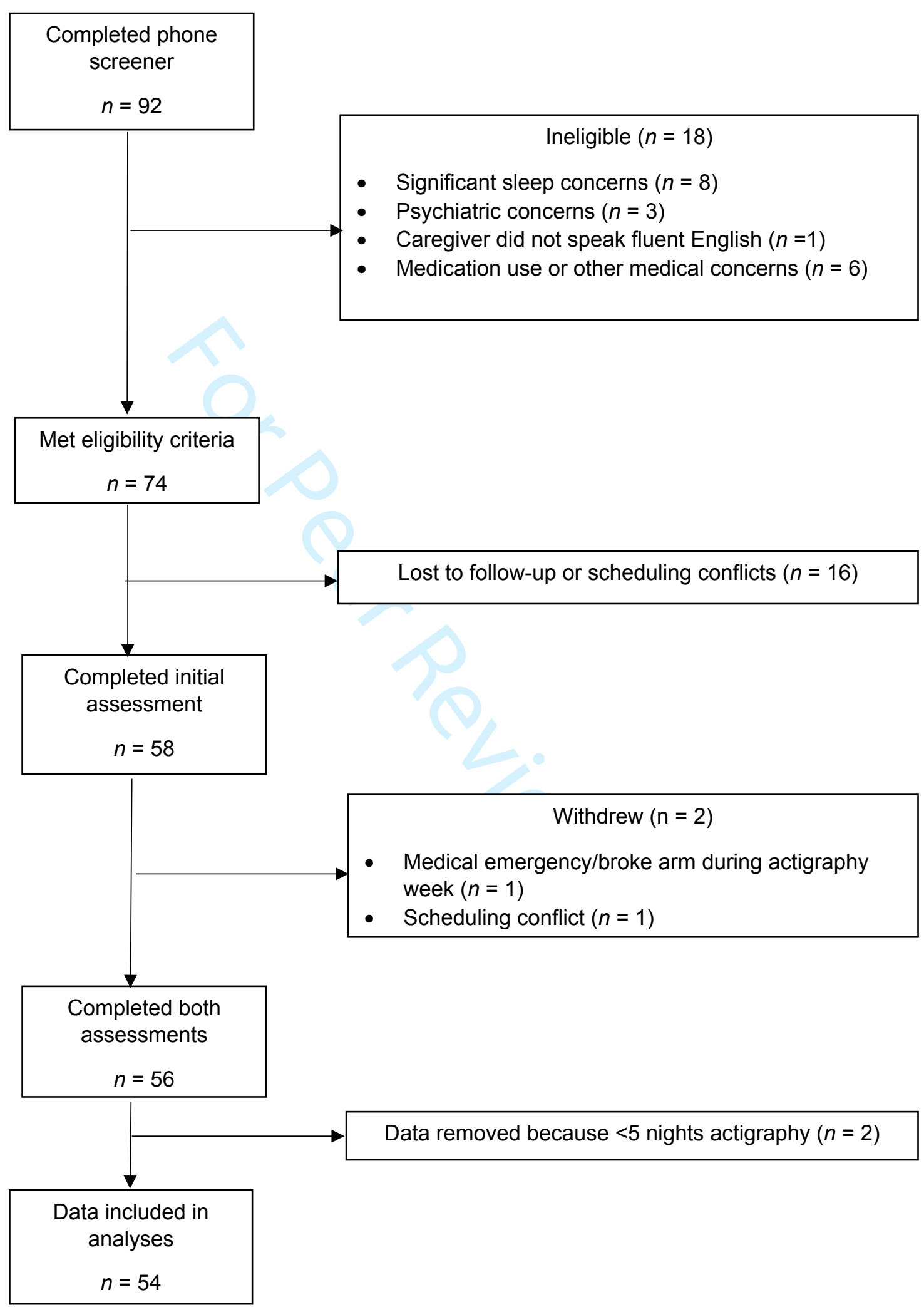


Supplemental File 2:

Principal Components Analysis for the Use of Situation Selection Questionnaire

A principal components analysis was conducted on the six items from the Use of Situation Selection Questionnaire (Webb et al., 2018) with orthogonal rotation (Varimax). The KaiserMeyer-Olkin measured provided verification of the sampling adequacy for analysis (overall KMO $=.74$; all $\mathrm{KMO}$ values for individual items ranged from .61-.79). Bartlett's test of sphericity suggested correlations between variables were adequate for conducting a $\mathrm{PCA}, X^{2}(15)=$ $116.92, p<.001$.

Results suggested a two-factor solution with eigenvalues $>1$. Together, these two factors contributed to $71.99 \%$ of the variance. Additional review of the scree plot and the component plot further supported a two-factor solution (see Figures 1 and 2). Table 1 shows the factor loadings after rotation. The clustering of items support Factor 1 as items referring to the approach of positive situations, whereas Factor 2 includes items representing the avoidance of negative situations.

Table 1

Factor Loadings for Principal Components Analysis

\begin{tabular}{lll}
\hline & Factor 1 & Factor 2 \\
\hline I select activities that help me to feel good. (SS_1) & .89 & -.08 \\
If a situation makes me feel good, then I try to stick around. (SS_2) & .87 & -.01 \\
I gravitate towards people, situations, and activities that put me in a good mood. (SS_3) & .74 & .39 \\
I keep doing something if it seems to be improving my mood. (SS_4) & .80 & .23 \\
I shy away from situations that might upset me. (SS_5) & .12 & .80 \\
I steer clear of people who put me in a bad mood. (SS_6) & .04 & .85 \\
\hline Eigenvalue & 2.94 & 1.38 \\
Variance Explained & $48.97 \%$ & $23.02 \%$ \\
\hline
\end{tabular}




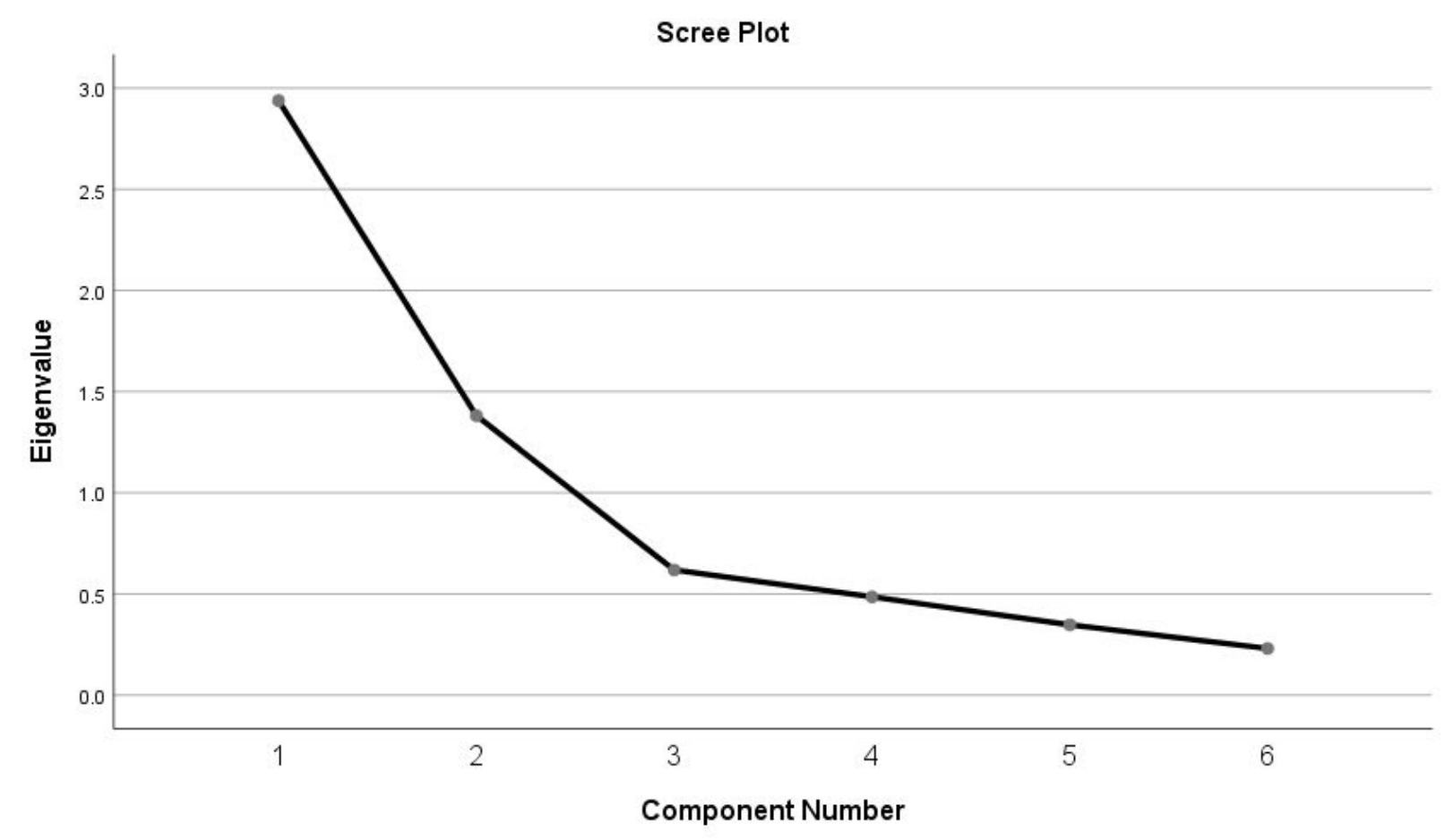

Figure 1. Scree plot for PCA on the Use of Situation Selection questionnaire.

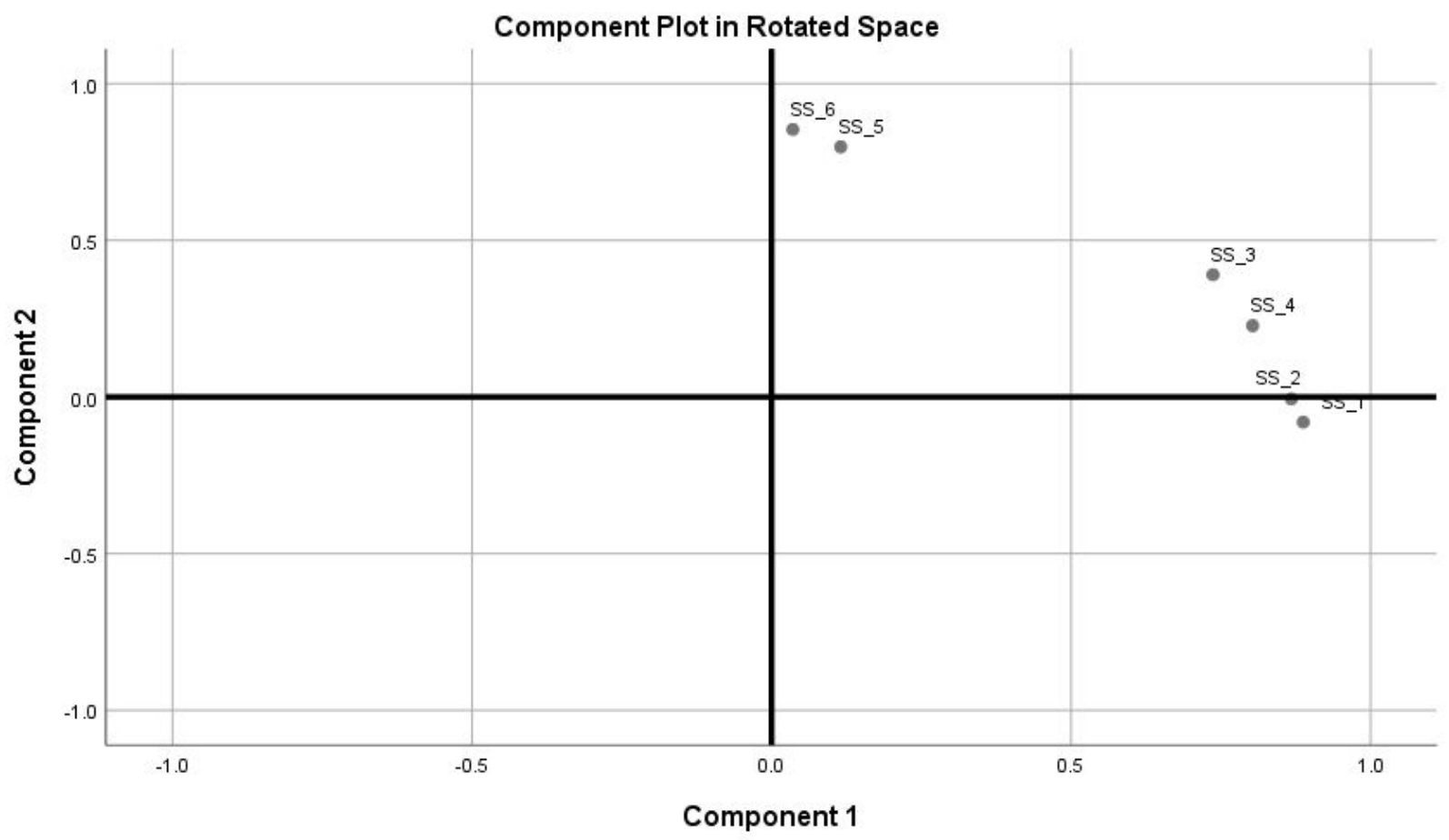

Figure 2. Component plot of the PCA on the Use of Situation Selection questionnaire. 